\title{
25 Research Square \\ Risk Factors and Microbiological Features of \\ Recurrent Escherichia coli Bloodstream Infection
}

\section{Yong Chan Kim}

Yonsei University Medical Center: Yonsei University Health System

\section{Heun Choi}

NHIC Ilsan Hospital: National Health Insurance Corporation Ilsan Hospital

\section{Young Ah Kim}

NHIC Ilsan Hospital: National Health Insurance Corporation Ilsan Hospital

\section{Yoon Soo Park ( $\square$ ysparkok2@yuhs.ac)}

Yonsei University Medical Center: Yonsei University Health System

\section{Young Hee Seo}

Yonsei University Medical Center: Yonsei University Health System

Hyukmin Lee

Yonsei University Medical Center: Yonsei University Health System

Kyungwon Lee

Yonsei University Medical Center: Yonsei University Health System

\section{Research}

Keywords: recurrent bloodstream infection, Escherichia coli, risk factors, whole genome sequencing

Posted Date: September 13th, 2021

DOI: https://doi.org/10.21203/rs.3.rs-860988/v1

License: (c) (1) This work is licensed under a Creative Commons Attribution 4.0 International License.

Read Full License 


\section{Abstract}

Background: Recurrent blood stream infection (BSI) is a significant complication associated with additional morbidity and mortality. We aim to understand the clinical risk factors and microbiological determinants in recurrent $E$. coli $\mathrm{BSI}$.

Methods: A retrospective study was conducted involving adult patients with $E$. coil $\mathrm{BSI}$ and its recurrence from January 2017 to December 2018. Recurrence was subdivided into early onset (4-30 days after initial BSI) and late onset (31-270 days after initial BSI). We evaluated risk factors for recurrent BSI and microbiological determinants of $E$. coli isolated from patients with recurrent BSI.

Results: Of 808 patients with E. coli BSI, 57 (6.31\%) had recurrence (29 of early onset and 28 of late onset) during study period. Data of 149 patients with single episode, whose samples were available for determining the sequence type 131 and extended-spectrum $\beta$-lactamase (ESBL) genotype, were selected for comparison. In recurrent BSI, liver cirrhosis, vascular catheter, and ESBL phenotype were more common, and the Charlson comorbidity index and sequential organ failure assessment score were higher than those of single episode BSI. Vascular catheter (odds ratio [OR], 4.588; 95\% confidence interval [Cl], 1.049-20.068), ESBL phenotype (OR, 2.037; 95\% Cl, 1.037-3.999) and sequential organ failure assessment score $\geq 9(\mathrm{OR}, 3.210 ; 95 \% \mathrm{Cl}, 1.359-7.581)$ were independent risk factors for recurrence. The proportion of the ESBL genotype was higher in early onset recurrent BSI (41.4\%) than in late onset recurrent $\mathrm{BSI}(25 \%)$ or single episode $\mathrm{BSI}(16.8 \%)$; the $E$. coli isolated from the former was resistant to most antimicrobial agents. Whole genome sequencing performed on 27 of the available ESBL-producing E. coli samples ( 11 from single episode, 11 from early onset recurrence, and 5 from late onset recurrence) demonstrated that various virulence factors, resistant genes, and plasmid types existed in isolates from all types of BSI. Among 28 patients of late onset recurrence, 8 episodes were available for pulsed-field gel electrophoresis analysis and $3(37.5 \%)$ were caused by an identical strain.

Conclusions: This study outlines risk factors contributing to the recurrence and microbiological features of $E$. coli causing recurrent $\mathrm{BSI}$, which may be helpful for healthcare providers.

\section{Introduction}

Escherichia coli is a gram-negative bacteria that is a part of normal gut microbiota but also cause blood stream infections (BSI) in humans [1]. For the last decade, E. coli has been the leading causative organism resulting in BSI in both community and hospital settings [2-4]. The proportion of BSI episodes caused by E. coli was $27.1 \%$, and the incidence rate was 48.0 per 100,000 person-year in high-income countries [5]. According a study conducted in England, BSI caused by E. coli has been increasing, and there was a 6\% increase in incidence between 2012-13 and 2013-14 [2].

E. coli is one of the most common pathogens isolated from recurrent BSI [6-8], and the rates of recurrence varied from 3.1\% [9] to 9.9\% [10]. Several factors are associated with recurrence of $E$. coli BSI in patients [6, 7, 9-11], such as hematologic malignancy, non-urinary tract infection, inadequate antibiotic 
therapy, and presence of vascular catheter. Antibiotic resistance in E. coli is also associated with an increase in recurrent BSI [12]. In particular, multidrug-resistant traits of extended-spectrum $\beta$-lactamase (ESBL)-producing $E$. coli hinder patients from receiving appropriate empirical antibiotic therapy and effective definite antibiotic therapy cause recurrent BSI [13]. Specific virulence factors of $E$. coli are associated with recurrence, because they prevent host-immune reactions resulting in delayed or failed clearance of pathogens [9].

Recurrence is a significant problem associated with E. coli BSI, as it may require re-admission to a hospital thereby adding to healthcare costs and can potentially increase the mortality rate. Therefore, it is important to investigate the prevalence, clinical characteristics, and microbiological features of recurrent BSI caused by $E$. coli. This information would be helpful for effectively planning patient management and follow-up after initial episode.

The aim of the current study was to determine risk factors for recurrent E. coli BSI in South Korea, where $E$. coli isolated from blood specimens exhibited high rates of resistance to several antimicrobial agents. In addition, we evaluated the molecular characteristics of $E$. coli causing recurrent $\mathrm{BSI}$, since the molecular epidemiology of recurrent $E$. coli has not yet been determined in this setting.

\section{Methods}

\section{Study design and population}

A retrospective study was performed at the National Health Insurance Service Ilsan Hospital in South Korea from January 2017 to December 2018. Among patients admitted to the hospital, adult patients (age $\geq 18$ years) who presented with positive blood culture for $E$. coli at least once were included in this study. Patients were followed up to 270 days after the initial episode of E.coli BSI. Patients who died or were transferred to another hospital within three days after initial detection of $E$. coli in the blood culture were excluded, as were patients who were expected to have recurrent bacteremia due to specific infection, such as infective endocarditis, mycotic aneurysm, and endovascular graft infection. All patients were included for analysis only once for each episode of BSI. Demographic and clinical variables were extracted through electronic medical records.

\section{Definition and classification of recurrent BSI}

Recurrent BSI was defined as a positive blood culture from a specimen collected 4 days or later after the first episode. Recurrence was subdivided into early onset (4-30 days after initial BSI) and late onset (31270 days after initial BSI). Type of infection were classified as community- or hospital-acquired. Community-acquired BSI was defined as an infection occurred within 48 hours after admission. Infections developed more than 48 hours after admission were defined as hospital-acquired BSI. Comorbidities were defined using the International Classification of Diseases, 10th revision. Charlson Comorbidity Index, which predicts the 10-year mortality in patients who may have a range of comorbid 
conditions [14], was used to quantify a patient's burden of disease. Prior antibiotics use was defined as use of antimicrobials for $\geq 3$ days in the previous 30 days. The primary focus of BSI was classified according to the Centers for Disease Control and Prevention/National Healthcare Safety Network surveillance criteria [15]. Empirical antibiotics were regarded to be appropriate if administered within one calendar day of culture, and the pathogen was susceptible to any of antibiotics used. Appropriate definite antibiotic therapy was defined as the administration of antibiotics based on the susceptibility, within one calendar day of the susceptibility results becoming available. We classified recurrence as a relapse when a strain of the second episode had an identical Pulsed-field gel electrophoresis (PFGE) pattern to that of the initial episode. Recurrence was considered as a reinfection when the PFGE pattern was different between strains of the initial and subsequent episodes.

\section{Microbiological analysis}

Blood culture was performed using BacT/ALERT FA/NA (BioMerieux Inc., Durham, NC, USA) with the incubation of blood bottles at $37^{\circ} \mathrm{C}$ for five days. Species were identified with MALDI Biotyper MALDI-TOF MS (Bruker Daltonik, Bremen, Germany). The antimicrobial susceptibility test was conducted using MicroScan WalkAway plus system (Beckman Coulter, Inc., West Sacramento, CA, USA) and MicroScan Neg Breakpoint Combo Type 44 panel (Siemens Healthcare Diagnostics, Inc., West Sacramento, CA, USA). The susceptibility results were interpreted using the Clinical and Laboratory Standards Institute guidelines [16].

We performed polymerase chain reaction (PCR)-sequencing of $025 \mathrm{~b}$ and 016 to determine the sequence type (ST) 131, and a DNA region corresponding to CTX-M, SHV, and TEM was sequenced to detect the genotype of ESBL. PFGE was performed on eight pairs of late onset recurrence to distinguish between relapse and reinfection. The patterns were analyzed using InfoQuest FP software (Bio-Rad) to generate a dendrogram based on the unweighted pair group method, with an arithmetic average (UPGMA) from the Dice coefficient with $1 \%$ band position tolerance and $0.5 \%$ optimization settings [17].

We evaluated the ST, virulence factor, plasmid type, and antimicrobial resistance gene of ESBLproducing $E$. coli using the whole genome sequencing (WGS). WGS was performed on ESBL-producing E. coli isolated from patients with recurrent BSI and single episode BSI. The DNA of freshly subcultured isolates was extracted using GenElute ${ }^{\mathrm{TM}}$ Bacterial Genomic DNA Kit (Sigma-Aldrich, St. Louis, MO), and 8 $\mu \mathrm{g}$ of input genomic DNA was used. The entire genomes of ESBL-producing $E$. coli isolates were sequenced using NextSeq 550 instrument (Illumina, San Diego, CA, USA), and sequences were assembled by Spades (version 3.11.1) and annotated by Prokka (version 1.13.7). The data of multilocus sequence typing (MLST), plasmid typing, virulence genes, and resistance genes were obtained from the web site of center for genomic epidemiology (www.genomicepidemiology.org). The virulence score was calculated as the sum of all virulence factors, as described by Johnson et al [18].

\section{Statistical analysis}


The results were presented as numbers with percentages for categorical variables and median with interquartile range (IQR) for continuous variables. The continuous variables were compared using the MannWhitney $U$ test. The chi-squared test was used to compare the categorical variables. Logistic regression analysis was performed to determine risk factors for recurrent BSI. Variables found to be significant in univariate analysis were included in a multivariate logistic regression analysis. Results were considered statistically significant at $P<0.05$. All statistical analyses were performed using SPSS 23.0 software (SPSS, Chicago, IL, USA).

\section{Results}

Clinical characteristics of patients with recurrent and single episode BSI caused by E. coli

A total of 808 patients experienced E. coli BSI during study period, and of them, 57 (6.31\%) patients had recurrent episodes. Clinical characteristics of 206 patients, 57 with recurrent episode and 149 whose samples were available for determining the ST 131 and ESBL genotype, with single episode were described in Table 1. Median age was 77 (IQR, 71-84) and 78 (IQR, 67-85) in the recurrent episode and single episode groups, respectively. Female biased sex ratio was observed (64.9\% in recurrent episode group and $71.1 \%$ in single episode group). The proportion of liver cirrhosis was higher in the recurrent episode group $(N=6,10.5 \%)$ than in the single episode group $(N=3,2.0 \%)$. Higher Charlson comorbidity index was detected in patients with recurrent episode (2, IQR, 0-4) than in those with single episode (1, IQR, 0-2). Patients who had vascular catheter were more common in recurrent episode than in single episode $(\mathrm{N}=6,10.5 \%$ vs $\mathrm{N}=3,2.0 \%)$. $E$. coli with the $\mathrm{ESBL}$ phenotype was more frequently isolated from samples of recurrent episode than single episode $(N=24,42.1 \%$ vs $N=37,24.8 \%)$. Sequential organ failure assessment (SOFA) score was higher in recurrent episode group (4, IQR, 2-8) than in single episode group (3, IQR, 1-6). Mortality rates did not significantly differ between both groups $(P>0.999)$. 
Table 1

Comparison of patients with recurrent E. coli BSI and single episode of $E$. coli BSI

\begin{tabular}{|c|c|c|c|c|}
\hline Variables & $\begin{array}{l}\text { Total } \\
(\mathrm{N}=206)\end{array}$ & $\begin{array}{l}\text { Single } \\
\text { episode } \\
(N=149)\end{array}$ & $\begin{array}{l}\text { Recurrent } \\
\text { episode } \\
(\mathrm{N}=57)\end{array}$ & $P$ \\
\hline Age, years & $\begin{array}{l}78(70- \\
84)\end{array}$ & $78(67-85)$ & $77(71-84)$ & 0.861 \\
\hline Gender, n (\%) & & & & 0.385 \\
\hline Female & $\begin{array}{l}143 \\
(69.42)\end{array}$ & $106(71.1)$ & $37(64.9)$ & \\
\hline Male & $63(30.6)$ & $43(28.9)$ & $20(35.1)$ & \\
\hline Hospital acquired infection, $\mathrm{n}(\%)$ & $90(43.7)$ & $64(43.0)$ & $26(45.6)$ & 0.731 \\
\hline Transferred case, n (\%) & & & & 0.894 \\
\hline Non-transferred & $114(55.3)$ & $83(55.7)$ & $31(54.4)$ & \\
\hline Long-term care facility & $17(8.3)$ & $13(8.7)$ & $4(7.0)$ & \\
\hline Acute care hospital & $37(18.0)$ & $25(16.8)$ & $12(21.1)$ & \\
\hline Clinic & $38(18.5)$ & $28(18.8)$ & $10(17.5)$ & \\
\hline \multicolumn{5}{|l|}{ Comorbidity, n (\%) } \\
\hline Diabetes mellitus & $56(27.2)$ & $41(27.5)$ & $15(26.3)$ & 0.862 \\
\hline Heart failure & $8(3.9)$ & $6(4.0)$ & $2(3.5)$ & $>.999$ \\
\hline Pulmonary disease & $4(1.9)$ & $3(2.0)$ & $1(1.8)$ & $>.999$ \\
\hline Chronic kidney disease & $23(11.2)$ & $15(10.1)$ & $8(14.0)$ & 0.419 \\
\hline Liver cirrhosis & $9(4.4)$ & $3(2.0)$ & $6(10.5)$ & 0.015 \\
\hline Malignancy & $40(19.4)$ & $25(16.8)$ & $15(26.3)$ & 0.122 \\
\hline Charlson comorbidity index & $1(0-3)$ & $1(0-2)$ & $2(0-4)$ & 0.013 \\
\hline Primary focus of bacteremia, $\mathrm{n}(\%)$ & & & & 0.114 \\
\hline Non-urinary tract infection & $66(32.0)$ & $43(28.9)$ & $23(40.4)$ & \\
\hline Urinary tract infection & $140(68.0)$ & $106(71.1)$ & $34(59.7)$ & \\
\hline $\begin{array}{l}\text { Polymicrobial blood stream infection, } n \\
(\%)\end{array}$ & $11(5.3)$ & $9(6.0)$ & $2(3.5)$ & 0.731 \\
\hline
\end{tabular}

BSI, bloodstream infection; ESBL, extended spectrum $\beta$-lactamase; SOFA, sequential organ failure assessment 


\begin{tabular}{|c|c|c|c|c|}
\hline Variables & $\begin{array}{l}\text { Total } \\
(\mathrm{N}=206)\end{array}$ & $\begin{array}{l}\text { Single } \\
\text { episode } \\
(N=149)\end{array}$ & $\begin{array}{l}\text { Recurrent } \\
\text { episode } \\
(\mathrm{N}=57)\end{array}$ & $P$ \\
\hline Prior antibiotic use, $\mathrm{n}(\%)$ & $180(87.4)$ & $132(88.6)$ & $48(84.2)$ & 0.397 \\
\hline $\begin{array}{l}\text { Appropriate empirical antibiotic use, } \mathrm{n} \\
(\%)\end{array}$ & $152(73.8)$ & $112(75.2)$ & $40(70.2)$ & 0.466 \\
\hline Appropriate definite antibiotic use, $n(\%)$ & $183(88.8)$ & $131(87.9)$ & $52(91.2)$ & 0.5 \\
\hline Duration of definite antibiotic use, days & $\begin{array}{l}14(12- \\
16)\end{array}$ & $14(11-15)$ & $14(13-17)$ & 0.126 \\
\hline \multicolumn{5}{|c|}{ Indwelling catheter in the previous 30 days, $n$ (\%) } \\
\hline Vascular catheter & $9(4.4)$ & $3(2.0)$ & $6(10.7)$ & 0.014 \\
\hline Urinary catheter & $128(62.1)$ & $95(63.8)$ & $33(57.9)$ & 0.438 \\
\hline E. coli with ESBL phenotype, $\mathrm{n}(\%)$ & $61(29.6)$ & $37(24.8)$ & $24(42.1)$ & 0.015 \\
\hline SOFA score & $3(1-7)$ & $3(1-6)$ & $4(2-8)$ & 0.035 \\
\hline 30-day mortality, n (\%) & $13(6.3)$ & $10(6.7)$ & $3(5.3)$ & $>.999$ \\
\hline
\end{tabular}

Fifty-seven recurrent $E$. coli BSI were subdivided into early onset recurrence (29) and late onset recurrence (28). Median time to recurrence since first episode was 6 days (IQR, 4-15.5) and 66.5 days (IQR, 44.3134.3) in early onset recurrence and late onset recurrence, respectively. Comparison between two groups is demonstrated in Supplementary Table 1.

\section{Risk factors for recurrent E. coli BSI}

Univariate logistic regression analysis demonstrated that liver cirrhosis, Charlson comorbidity index $\geq 3$, vascular catheter, E. coli with ESBL phenotype, and SOFA score $\geq 9$ were associated with recurrent $E$. coli BSI (Table 2). In multivariate analysis, independent risk factors for recurrent bacteremia were vascular catheter (odds ratio [OR], 4.588; 95\% confidence interval [Cl], 1.049-20.068), E. coli with ESBL phenotype (OR, 2.037; 95\% Cl, 1.037-3.999), and SOFA score $\geq 9$ (OR, 3.210; 95\% Cl, 1.359-7.581). 
Table 2

Risk factors for recurrent E. coli BSI

\begin{tabular}{|c|c|c|c|c|}
\hline \multirow[t]{2}{*}{ Variables } & \multicolumn{2}{|c|}{ Univariate analysis } & \multicolumn{2}{|c|}{ Multivariate analysis } \\
\hline & OR $(95 \% \mathrm{Cl})$ & $\mathrm{P}$ & OR $(95 \% \mathrm{Cl})$ & $P$ \\
\hline Age $\geq 65$ & $\begin{array}{l}1.577(0.703- \\
3.538)\end{array}$ & 0.269 & $\begin{array}{l}1.579(0.663- \\
3.763)\end{array}$ & 0.302 \\
\hline \multicolumn{5}{|l|}{ Gender } \\
\hline Female & Reference & & Reference & \\
\hline Male & $\begin{array}{l}1.332(0.696- \\
2.550)\end{array}$ & 0.386 & $\begin{array}{l}1.098(0.533- \\
2.265)\end{array}$ & 0.799 \\
\hline Hospital acquired infection & $\begin{array}{l}1.114(0.603- \\
2.058)\end{array}$ & 0.731 & & \\
\hline
\end{tabular}

Transferred case

Non-transferred

Reference

Long-term care facility

$0.824(0.25-2.719) \quad 0.751$

Acute care hospital

1.285 (0.576- $\quad 0.54$

2.867)

Clinic

$0.956(0.416-\quad 0.916$

2.196)

Comorbidity

Diabetes mellitus

$0.941(0.472-$

1.877)

0.862

Heart failure

$0.867(0.17-4.424) \quad 0.863$

Pulmonary disease

$\begin{array}{ll}0.869(0.089- & 0.904 \\ 8.531) & \end{array}$

Chronic kidney disease
$1.459(0.582-$
0.42
3.654)

Liver cirrhosis

$5.725(1.381-$ 23.735)

Cancer

$1.772(0.854-$

0.124

3.675)

Charlson comorbidity index $\geq 3$

$1.973(1.028-$

0.041

1.591 (0.791-

3.199)

0.193

Primary focus of bacteremia

$\mathrm{BSI}$, bloodstream infection; $\mathrm{OR}$, odd ratio; $\mathrm{Cl}$, confidence interval, $\mathrm{ESBL}$, extended spectrum $\beta$ lactamase; SOFA, sequential organ failure assessment 


\begin{tabular}{|c|c|c|c|c|}
\hline Variables & \multicolumn{2}{|l|}{ Univariate analysis } & \multicolumn{2}{|c|}{ Multivariate analysis } \\
\hline Non-urinary tract infection & \multicolumn{4}{|l|}{ Reference } \\
\hline Urinary tract infection & $0.6(0.317-1.134)$ & 0.116 & & \\
\hline Polymicrobial bloodstream infection & $\begin{array}{l}0.566(0.118- \\
2.702)\end{array}$ & 0.475 & & \\
\hline $\begin{array}{l}\text { Antibiotic use } \leq 1 \text { month before } \\
\text { episode }\end{array}$ & $\begin{array}{l}1.456(0.608- \\
3.485)\end{array}$ & 0.399 & & \\
\hline Appropriate empirical antibiotic use & $\begin{array}{l}0.777(0.394- \\
1.532)\end{array}$ & 0.467 & & \\
\hline Appropriate definite antibiotic use & $\begin{array}{l}1.429(0.504- \\
4.049)\end{array}$ & 0.502 & & \\
\hline Duration of definite antibiotic use & $\begin{array}{l}1.021(0.995- \\
1.047)\end{array}$ & 0.109 & & \\
\hline \multicolumn{5}{|l|}{ Indwelling catheter in 30 days } \\
\hline Vascular catheter & $\begin{array}{l}5.84(1.408- \\
24.222)\end{array}$ & 0.015 & $\begin{array}{l}4.588(1.049- \\
20.068)\end{array}$ & 0.043 \\
\hline Urinary catheter & $\begin{array}{l}0.782(0.419- \\
1.457)\end{array}$ & 0.438 & & \\
\hline Tracheal tube & $0.867(0.17-4.424)$ & 0.863 & & \\
\hline Nasogastric tube & $\begin{array}{l}1.456(0.608- \\
3.485)\end{array}$ & 0.399 & & \\
\hline E. coli with ESBL phenotype & $\begin{array}{l}2.201(1.156- \\
4.191)\end{array}$ & 0.016 & $\begin{array}{l}2.037 \text { (1.037- } \\
3.999)\end{array}$ & 0.039 \\
\hline SOFA score $\geq 9$ & $\begin{array}{l}3.406(1.487- \\
7.804)\end{array}$ & 0.004 & $\begin{array}{l}3.210(1.359- \\
7.581)\end{array}$ & 0.008 \\
\hline
\end{tabular}

\section{Molecular epidemiology and antimicrobial resistance of E. coli}

Among 206 isolates tested, E. coli ST131 clone was found in $53(25.7 \%)$, comprising ST131-025 ( N = 47) and ST131-016 ( $=6$ ) (Table 3). ST131 strains were more frequently observed in early onset recurrent episode $(N=12,41.4 \%)$ than in the late onset recurrent episode $(N=7,25.0 \%)$ and single episode $(N=34$, $22.8 \%)$. E. coli with ESBL genotype was more commonly observed in early onset recurrent episode $(\mathrm{N}=$ $12,41.4 \%)$ than in late onset recurrent episode $(N=7,25 \%)$ or in single episode $B S I(N=25,16.8 \%)$. CTXM-15 ( $N=25,56.8 \%)$ was the most common ESBL genotype, followed by CTX-M-14 ( $N=11,25 \%)$. 
Table 3

The molecular epidemiology of E. coli isolated from patients with BSI

\begin{tabular}{|c|c|c|c|}
\hline & $\begin{array}{l}\text { Single episode } \\
\text { blood stream infection } \\
(N=149)\end{array}$ & $\begin{array}{l}\text { Early onset recurrent } \\
\text { blood stream infection } \\
(\mathrm{N}=29)\end{array}$ & $\begin{array}{l}\text { Late onset recurrent } \\
\text { blood stream infection } \\
(\mathrm{N}=28)\end{array}$ \\
\hline ST131, n (\%) & $34(22.8)$ & $12(41.4)$ & $7(25)$ \\
\hline 025 & 29 (19.5) & $12(41.4)$ & $6(21.4)$ \\
\hline 016 & $5(3.4)$ & 0 & $1(3.6)$ \\
\hline ESBL genotype, n (\%) & $25(16.8)$ & $12(41.4)$ & $7(25)$ \\
\hline CTX-M-3 & 0 & 0 & $1(3.6)$ \\
\hline CTX-M-14 & $5(3.4)$ & $3(10.3)$ & $3(10.7)$ \\
\hline CTX-M-15 & $17(11.4)$ & $5(17.2)$ & $3(10.7)$ \\
\hline CTX-M-27 & $2(1.3)$ & $4(13.8)$ & 0 \\
\hline CTX-M-55 & $1(0.7)$ & 0 & 0 \\
\hline
\end{tabular}

E. coli isolated from early onset recurrent BSI had higher resistance rates to most antimicrobial agents than that isolated from late onset recurrent BSI or single episode BSI (Fig. 1). Resistance rates to piperacillin/tazobactam, cefoxitin, minocycline, chloramphenicol, fosfomycin, and tigecycline were very low in all the groups. A strain resistant to amikacin, colistin, and carbapenem was not identified in this study.

\section{WGS of ESBL-producing E. coli}

Twenty-seven of ESBL-producing E. coli were available for WGS; 11 from single episodes, 11 from early onset recurrent episodes, and 5 from late onset recurrent episodes. Various antimicrobial resistance genes, plasmid types, and virulence factors were detected isolated from all type of BSI (Table 4). There were resistance genes to $\beta$-lactam (b/a), streptomycin (str), aminoglycoside (aac, aad), macrolide ( $m p h)$, quinolone ( $\left.\operatorname{aac}\left(6^{\prime}\right) l b-c r\right)$, trimethoprim-sulfamethoxazole ( $d f r$, sul), tetracycline (tet), and chloramphenicol (cat). IncFIA, IncFIB, and IncFIl were commonly detected plasmid types, but there was no dominant plasmid type. Variable virulence genes were detected, and gad was the most common virulent gene, followed by iss, iha, and sat. The median scores of virulence were 5 (IQR, 3-7), 4.5 (IQR, 1-6), and 5 (IQR, 2-8) for early onset recurrent, late onset recurrent, and single episode BSI, respectively. 
Table 4

Whole genome sequencing data of ESBL-producing E. coli isolated from patients with BSI

\begin{tabular}{|c|c|c|c|c|c|}
\hline No & Episode & ST & Resistance gene & Plasmid & $\begin{array}{l}\text { Virulence } \\
\text { gene }\end{array}$ \\
\hline $\begin{array}{l}\text { EC } \\
0049\end{array}$ & Single & 131 & $\begin{array}{l}\operatorname{aac}(3)-I l a, \operatorname{catB} 3, \operatorname{aac}\left(6^{\prime}\right) l b-c r, \\
\text { bla }{ }_{\text {OXA-1 }}, \text { bla }\end{array}$ & Not detected & $\begin{array}{l}\text { nfaE, iha, } \\
\text { gad, } \\
\text { cnf1, iss, } \\
\text { sat }\end{array}$ \\
\hline $\begin{array}{l}E C \\
0075\end{array}$ & Single & 131 & $b / a_{\mathrm{CTX}-\mathrm{M}-27}$ & $\begin{array}{l}\text { IncFIA, } \\
\text { IncFIB(AP001918), } \\
\text { IncFIB(pLF82- } \\
\text { PhagePlasmid), IncFII }\end{array}$ & $\begin{array}{l}\text { sat, iha, } \\
\text { iss, gad }\end{array}$ \\
\hline $\begin{array}{l}\text { EC } \\
0131\end{array}$ & Single & 131 & $\begin{array}{l}\text { aacA4, catB3, } \operatorname{aac}\left(6^{\prime}\right) l b-c r \\
\text { bla }_{\mathrm{CTX}-\mathrm{M}-15}, \text { bla } \mathrm{O}_{\mathrm{OXA}-1}\end{array}$ & $\begin{array}{l}\text { Col(BS512), IncFIA, } \\
\text { IncFIB(AP001918) }\end{array}$ & iss, gad \\
\hline $\begin{array}{l}\text { EC } \\
0306\end{array}$ & Single & 131 & $\begin{array}{l}\text { aadA5, } m p h(A), \text { sul1, dfrA17, } \\
\text { catB3, bla } \mathrm{CTX}-\mathrm{M}-15, \text { bla } \mathrm{OXX}-1 \\
\operatorname{aac}\left(6^{\prime}\right) / \mathrm{lb}-\mathrm{cr}\end{array}$ & $\begin{array}{l}\text { Col(BS512), Col156, } \\
\text { IncFIA, } \\
\text { IncFIB(AP001918) }\end{array}$ & $\begin{array}{l}\text { sat, } \\
\text { senB, } \\
\text { iha, iss, } \\
\text { gad }\end{array}$ \\
\hline $\begin{array}{l}E C \\
0364\end{array}$ & Single & 131 & 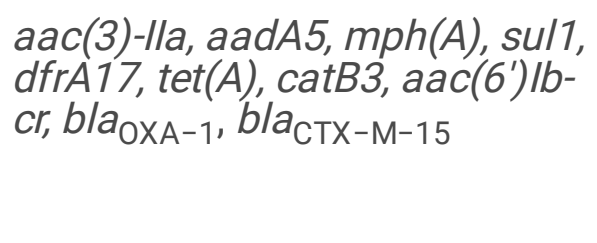 & $\begin{array}{l}\text { Col156, } \\
\text { IncFIA,IncFIB(AP001918) }\end{array}$ & $\begin{array}{l}\text { cnf1, iha, } \\
\text { gad, } \\
n f a E \text {, iss, } \\
\text { celb, } \\
\text { senB, sat }\end{array}$ \\
\hline $\begin{array}{l}\text { EC } \\
0436\end{array}$ & Single & 131 & 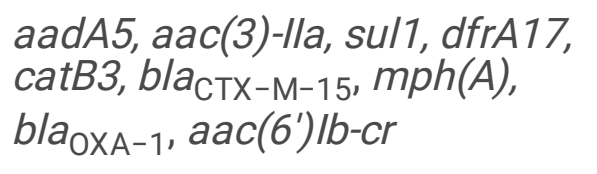 & $\begin{array}{l}\text { Col(BS512), IncFIA, } \\
\text { IncFIB(AP001918) }\end{array}$ & $\begin{array}{l}\text { sat, iha, } \\
\text { iss, gad }\end{array}$ \\
\hline $\begin{array}{l}\mathrm{EC} \\
0500\end{array}$ & Single & 131 & $\begin{array}{l}\operatorname{aad} A 5, \operatorname{aac}(3)-I l a, \text { sul1, dfrA17, } \\
\text { tet(A), catB3, bla } \mathrm{CTX-M-15} \\
m p h(A), \operatorname{bla} a_{0 X \mathrm{X}-1}, \operatorname{aac}\left(6^{\prime}\right) l b-c r\end{array}$ & $\begin{array}{l}\text { IncFIA, } \\
\text { IncFIB(AP001918) }\end{array}$ & $\begin{array}{l}\text { cnf1, iha, } \\
\text { gad, } \\
\text { nfaE, iss, } \\
\text { senB, sat }\end{array}$ \\
\hline $\begin{array}{l}\text { EC } \\
0530\end{array}$ & Single & 131 & $\begin{array}{l}\text { strA, strB, aac(3)-Ila, aadA5, } \\
\text { mph }(A), \operatorname{sul} 1, \text { sul2, dfrA17, } \\
\text { tet }(A), \text { catB3, bla }{ }_{\mathrm{CTX}-\mathrm{M}-15} \\
\text { bla }_{\mathrm{OXA}-1}, \operatorname{aac}\left(6^{\prime}\right) I b-c r\end{array}$ & $\begin{array}{l}\text { Col156, IncFIA, } \\
\text { IncFIB(AP001918), } \\
\text { IncFII(pRSB107) }\end{array}$ & $\begin{array}{l}\operatorname{sen} B, \\
\operatorname{ast} A, \text { iss, } \\
\operatorname{gad}\end{array}$ \\
\hline $\begin{array}{l}\mathrm{EC} \\
0745\end{array}$ & Single & 131 & $\begin{array}{l}\operatorname{aac}(3)-1 / a, c a t B 3, b / a_{\mathrm{CTX}-\mathrm{M}-15} \\
\text { bla }\end{array}$ & $\begin{array}{l}\text { IncFIA, } \\
\text { IncFIB(AP001918) }\end{array}$ & $\begin{array}{l}\text { nfaE, iha, } \\
\text { gad, } \\
\text { cnf1, iss, } \\
\text { sat }\end{array}$ \\
\hline $\begin{array}{l}\text { EC } \\
1064\end{array}$ & Single & 744 & $\begin{array}{l}\text { tet }(A), \text { tet }(B), \text { catA2, dfrA14, } \\
\text { bla }{ }_{\mathrm{CTX}-\mathrm{M}-55, \text { aac(3)-Ila }}\end{array}$ & $\begin{array}{l}\text { IncFIB(AP001918), } \\
\text { IncFIC(FII), IncX4 }\end{array}$ & cma, gad \\
\hline
\end{tabular}




\begin{tabular}{|c|c|c|c|c|c|}
\hline No & Episode & ST & Resistance gene & Plasmid & $\begin{array}{l}\text { Virulence } \\
\text { gene }\end{array}$ \\
\hline $\begin{array}{l}\mathrm{EC} \\
1346\end{array}$ & Single & 1193 & $\begin{array}{l}\text { strA, strB, aadA5, mph }(A), \text { sul1, } \\
\text { sul2, dfrA17, tet }(A), \text { bla }{ }_{\mathrm{CTX}-\mathrm{M}-27}\end{array}$ & $\begin{array}{l}\text { Col(BS512), Col156, } \\
\text { IncFIA, } \\
\text { IncFIB(AP001918) }\end{array}$ & $\begin{array}{l}\text { cnf1, } \\
\text { ireA, iha, } \\
\text { gad, vat, } \\
\text { senB, sat }\end{array}$ \\
\hline $\begin{array}{l}\mathrm{EC} \\
0295\end{array}$ & $\begin{array}{l}\text { Early } \\
\text { onset } \\
\text { recurrent }\end{array}$ & 131 & $\begin{array}{l}\text { aac(3)-Ild, blaTEM-1B, } \\
\text { bla } a_{\mathrm{CTX}-\mathrm{M}-14}\end{array}$ & $\begin{array}{l}\text { IncFIA, } \\
\text { IncFIB(AP001918) }\end{array}$ & $\begin{array}{l}\text { sat, cnf1, } \\
\text { iha, iss, } \\
\text { gad }\end{array}$ \\
\hline $\begin{array}{l}\mathrm{EC} \\
0355\end{array}$ & $\begin{array}{l}\text { Early } \\
\text { onset } \\
\text { recurrent }\end{array}$ & 95 & $\begin{array}{l}\operatorname{aadA5,} \text { aac(3)-Ild, sul1, dfrA17, } \\
\text { bla }_{\mathrm{CTX}-\mathrm{M}-14,} \mathrm{mph}(A)\end{array}$ & $\begin{array}{l}\text { Col156, IncB/O/K/Z, } \\
\text { IncFIB(AP001918), } \\
\text { IncFII(29) }\end{array}$ & $\begin{array}{l}\text { vat, ireA, } \\
\text { gad, iss, } \\
\text { sen } B\end{array}$ \\
\hline $\begin{array}{l}\text { EC } \\
0511\end{array}$ & $\begin{array}{l}\text { Early } \\
\text { onset } \\
\text { recurrent }\end{array}$ & 131 & $\begin{array}{l}\text { strA, strB, aadA5, mph }(A), \text { sul1, } \\
\text { sul2, } \operatorname{dfr} A 17, \operatorname{tet}(A), \text { bla } \mathrm{CTX}-\mathrm{M}-27\end{array}$ & $\begin{array}{l}\text { Col(KPHS6), IncFIA, } \\
\text { IncFIB(AP001918), } \\
\text { IncFII(pRSB107) }\end{array}$ & $\begin{array}{l}\text { sat, iss, } \\
\text { gad }\end{array}$ \\
\hline $\begin{array}{l}\text { EC } \\
0578\end{array}$ & $\begin{array}{l}\text { Early } \\
\text { onset } \\
\text { recurrent }\end{array}$ & 131 & 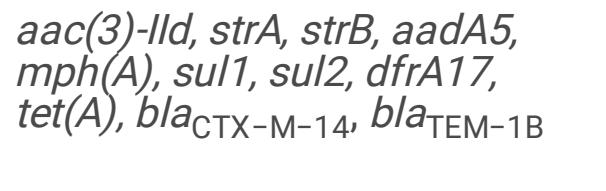 & $\begin{array}{l}\text { Col156, IncFIA, } \\
\text { IncFIB(AP001918), } \\
\text { IncFII(pRSB107) }\end{array}$ & $\begin{array}{l}\text { nfaE, iha, } \\
\text { gad, } \\
\text { cnf1, iss, } \\
\text { senB, sat }\end{array}$ \\
\hline $\begin{array}{l}\mathrm{EC} \\
0632\end{array}$ & $\begin{array}{l}\text { Early } \\
\text { onset } \\
\text { recurrent }\end{array}$ & 131 & $b / a_{\mathrm{CTX}-\mathrm{M}-27}$ & $\begin{array}{l}\text { Col156, IncFIA, } \\
\text { IncFIB(AP001918), } \\
\text { IncFII(pRSB107), Incl1- } \\
\text { I(Gamma) }\end{array}$ & $\begin{array}{l}\operatorname{sen} B, \text { iss, } \\
\text { gad }\end{array}$ \\
\hline $\begin{array}{l}\text { EC } \\
1132\end{array}$ & $\begin{array}{l}\text { Early } \\
\text { onset } \\
\text { recurrent }\end{array}$ & 1193 & $m p h(A), b / a^{\mathrm{CTX}-\mathrm{M}-15}$ & $\begin{array}{l}\text { Col(BS512), IncFIA, } \\
\text { IncFIB(APO01918), } \\
\text { IncFII(pHN7A8) }\end{array}$ & $\begin{array}{l}\text { sat, vat, } \\
\text { iha, } \\
\text { senB, } \\
\text { gad }\end{array}$ \\
\hline $\begin{array}{l}\text { EC } \\
1286\end{array}$ & $\begin{array}{l}\text { Early } \\
\text { onset } \\
\text { recurrent }\end{array}$ & 131 & $\begin{array}{l}\text { aac(3)-Ila, aadA5, mph(A), sul1, } \\
\text { dfrA17, tet }(A), \text { catB3, aac }\left(6^{\prime}\right) l b- \\
\text { cr, bla }\end{array}$ & $\begin{array}{l}\text { IncFIA, } \\
\text { IncFIB(AP001918), Incl1- } \\
\text { I(Gamma) }\end{array}$ & $\begin{array}{l}\text { nfaE, iha, } \\
\text { gad, } \\
\text { cnf1, iss, } \\
\text { senB, sat }\end{array}$ \\
\hline $\begin{array}{l}\text { EC } \\
1317\end{array}$ & $\begin{array}{l}\text { Early } \\
\text { onset } \\
\text { recurrent }\end{array}$ & 131 & $\begin{array}{l}\operatorname{aac}(3)-1 / a, \operatorname{tet}(A), \text { catB3, } \\
\operatorname{aac}\left(6^{\prime}\right) I b-c r, \text { bla }{ }_{0 X A-1} \\
\text { bla }_{\mathrm{CTX}-\mathrm{M}-15}\end{array}$ & $\begin{array}{l}\text { IncB/O/K/Z, IncFIA, } \\
\text { IncFIB(AP001918) }\end{array}$ & $\begin{array}{l}\text { sat, cnf1, } \\
\text { iha, iss, } \\
\text { gad }\end{array}$ \\
\hline $\begin{array}{l}\text { EC } \\
1398\end{array}$ & $\begin{array}{l}\text { Early } \\
\text { onset } \\
\text { recurrent }\end{array}$ & 131 & 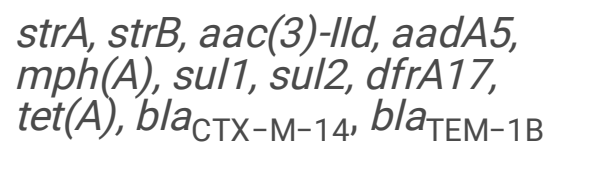 & $\begin{array}{l}\text { Col156, IncFIA, } \\
\text { IncFIB(AP001918), } \\
\text { IncFII(pRSB107) }\end{array}$ & $\begin{array}{l}\text { nfaE, iha, } \\
\text { cnf1, } \\
\text { gad, iss, } \\
\text { senB, sat }\end{array}$ \\
\hline $\begin{array}{l}\text { EC } \\
1508\end{array}$ & $\begin{array}{l}\text { Early } \\
\text { onset } \\
\text { recurrent }\end{array}$ & 131 & 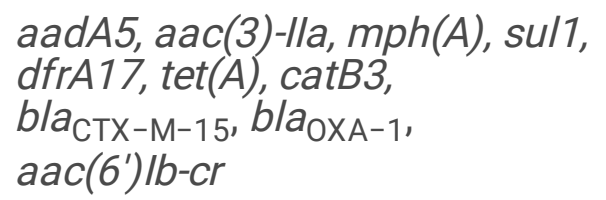 & $\begin{array}{l}\text { IncFIA, } \\
\text { IncFIB(AP001918) }\end{array}$ & $\begin{array}{l}\text { cnf1, iha, } \\
\text { gad, } \\
\text { nfaE, iss, } \\
\text { senB, sat }\end{array}$ \\
\hline
\end{tabular}

ESBL, extended spectrum $\beta$-lactamase; BSI, bloodstream infection; ST, sequence type 


\begin{tabular}{|c|c|c|c|c|c|}
\hline No & Episode & ST & Resistance gene & Plasmid & $\begin{array}{l}\text { Virulence } \\
\text { gene }\end{array}$ \\
\hline $\begin{array}{l}\text { EC } \\
1631\end{array}$ & $\begin{array}{l}\text { Early } \\
\text { onset } \\
\text { recurrent }\end{array}$ & 131 & $\begin{array}{l}\text { strA, strB, aadA5, mph(A), sul1, } \\
\text { sul2, } \operatorname{dfr} A 17, \operatorname{tet}(A), \text { bla } \mathrm{CTX-M-27}\end{array}$ & $\begin{array}{l}\text { IncFIA, } \\
\text { IncFIB(AP001918), } \\
\text { IncFII(pRSB107) }\end{array}$ & $\begin{array}{l}\text { senB, } \\
\text { ireA, iha, } \\
\text { iss, gad }\end{array}$ \\
\hline $\begin{array}{l}\mathrm{EC} \\
0151\end{array}$ & $\begin{array}{l}\text { Late } \\
\text { onset } \\
\text { recurrent }\end{array}$ & 131 & $\begin{array}{l}\text { aac(3)-Ila, catB3, aac }\left(6^{\prime}\right) / b-c r, \\
\text { bla }_{\text {OXA-1 }}, \text { bla }_{\mathrm{CTX}-\mathrm{M}-15}, \operatorname{tet}(A)\end{array}$ & $\begin{array}{l}\text { Col156, IncFIA, } \\
\text { IncFIB(AP001918) }\end{array}$ & $\begin{array}{l}\text { cnf1, iha, } \\
\text { nfaE, } \\
\text { gad, iss, } \\
\text { sat }\end{array}$ \\
\hline $\begin{array}{l}\mathrm{EC} \\
1254\end{array}$ & $\begin{array}{l}\text { Late } \\
\text { onset } \\
\text { recurrent }\end{array}$ & 648 & $\begin{array}{l}\operatorname{aac}(3)-1 / a, \operatorname{aad} A 5, \operatorname{mph}(A), \text { sul1, } \\
\operatorname{dfrA17} \text {, tet(B), bla }{ }_{\text {CTX-M-14 }}\end{array}$ & $\begin{array}{l}\text { Col(MG828), IncFIA, } \\
\text { IncFIB(AP001918), } \\
\text { IncFII(pRSB107) }\end{array}$ & $\begin{array}{l}\text { eilA, IpfA, } \\
\text { air }\end{array}$ \\
\hline $\begin{array}{l}\mathrm{EC} \\
1256\end{array}$ & $\begin{array}{l}\text { Late } \\
\text { onset } \\
\text { recurrent }\end{array}$ & 131 & 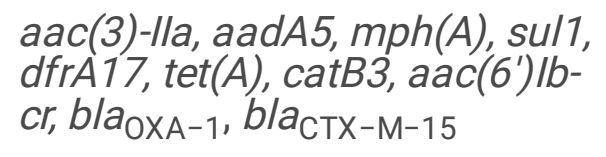 & $\begin{array}{l}\text { Col156, ColpVC, IncFIA, } \\
\text { IncFIB(AP001918) }\end{array}$ & $\begin{array}{l}\text { cnf1, iha, } \\
\text { gad, iss, } \\
\text { senB, sat }\end{array}$ \\
\hline $\begin{array}{l}\text { EC } \\
1448\end{array}$ & $\begin{array}{l}\text { Late } \\
\text { onset } \\
\text { recurrent }\end{array}$ & 131 & $\begin{array}{l}\text { aac(3)-Ild, strA, strB, aadA5, } \\
m p h(A), \text { sul1, sul2, dfrA17, } \\
\text { tet }(A), \text { bla } \mathrm{CTX}-\mathrm{M}-3, \text { bla } \text { TEM-1B }^{2}\end{array}$ & IncFIB(AP001918), IncFII & iha \\
\hline $\begin{array}{l}\text { EC } \\
1563\end{array}$ & $\begin{array}{l}\text { Late } \\
\text { onset } \\
\text { recurrent }\end{array}$ & 131 & 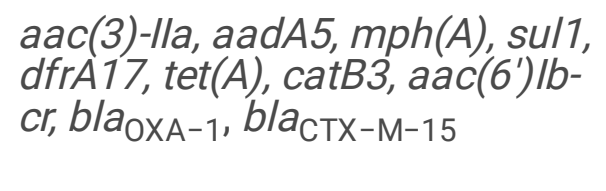 & $\begin{array}{l}\text { Col156, IncFIA, } \\
\text { IncFIB(AP001918) }\end{array}$ & $\begin{array}{l}\text { cnf1, iha, } \\
\text { gad, } \\
n f a E \text {, iss, } \\
\operatorname{sen} B \text {, sat }\end{array}$ \\
\hline
\end{tabular}

\section{Relapses versus reinfections in late onset recurrent blood stream infection}

PFGE was performed on eight paired isolates of the initial and subsequent episodes in late onset recurrent BSI (Fig. 2). Three pairs (28/28 - 2, 36/36 - 2, and 48/48 - 2) of eight paired isolates had identical PFGE patterns, ST type, and ESBL genotype, which suggested relapse rather than reinfection.

\section{Discussion}

This study demonstrated that the recurrent $E$. coli BSI was not uncommon, and the rate of early onset recurrence was similar to that of late onset recurrence. The risk factors for recurrent $E$. coli BSI were vascular catheter, ESBL phenotype, and SOFA score $\geq 9$. Multidrug-resistant $E$. coli of ST131 clone producing ESBL were more common in early onset recurrent BSI than in late onset recurrent BSI or single episode BSI. PFGE analysis suggested that a few cases of late onset recurrent BSI were attributed to relapse rather than reinfection. WGS demonstrated that various resistant genes and plasmid types were present in $\mathrm{ESBL}$-producing E. coli causing recurrent BSI.

Recurrence is a serious complication associated with BSI, and patients may have recurrent BSI. The rate of recurrent BSI varies and depends on the causative pathogens, definition of recurrence, and follow-up 
duration, but it is estimated to be $5-12 \%$ for Staphylococcus aureus [19-21], 2.3\% for Streptococcus pneumonia [22], 5.6\% for Acinetobacter baumannii [23], and 15.7\% for Salmonella species [24]. In this study, we found that in $6.3 \%$ of patients with $E$. coli BSI, the infection recurred. Our study also showed additional data for recurrence rate subdivided into early onset (3.6\%) and late onset (3.5\%) groups. To date, there is limited information on the recurrence rate changing with regard to time after the first episode, which may be clinically relevant and can help plan an appropriate follow-up for $E$. coli BSI patients.

We identified that ESBL-producing E. coli was an important risk factor for recurrent BSI. Maslow et al. showed that impaired host defense system was associated with recurrent episodes [9]. According to study by Sanz-Garci'a et al., male sex, presence of hematologic malignancy, inadequate antibiotic use, and extra-urinary source of BSI were independent risk factors for recurrence [11]. As these studies were conducted before the widespread infection with ESBL-producing E. coli [25-27], they could not evaluate the association of ESBL-producing $E$. coli with recurrence. Patients with BSI caused by ESBL-producing $E$. coli may have a greater chance of subsequent recurrent BSI due to multidrug-resistant traits of ESBLproducing E. coli. There is some evidence to support our results that antibiotic resistance of $E$. coli isolated from initial episode increases the risk of recurrent BSI [12, 13].

Recurrent BSI can be caused by infection with identical (relapse) or different (reinfection) isolates which is usually determined by molecular typing. PFGE analysis was performed on available $E$. coli isolated from late onset recurrence. Relapse was less common than reinfection but was observed at a substantial rate. Reinfection is associated with a higher degree of severity of the BSI-induced illness that may be difficult to modify and/or prevent [6]. In contrast, relapse may occur from the remnant foci of infection, undertreated during the treatment regimen of the prior episodes. This implies that appropriate therapy, such as elimination of residual infection foci, may reduce the rate of relapse.

The prevalence of ESBL-producing E. coli BSI has increased over the past decade owing to the widespread infection with the ST 131 clone containing CTX-M-15 [25-27]. To date, limited data are available for the rate of ESBL-producing $E$. coli in recurrent BSI. We showed the rate of ESBL-producing E. coli (33.3\%) and the most common ESBL genotype (CTX-M-15) in recurrent BSI. Multiple virulence factors may be involved in the survival of ESBL-producing $E$. coli resulting in recurrence. In our study, gad that is associated with protection of cells in acidic environment was the most prevalent virulence gene. Another virulence genes observed in $\mathrm{ESBL}$-producing $E$. coli causing recurrence were iss, iha, and sat, which are involved in immune evasion, adhesion, and protease production, respectively $[28,29]$.

This study has some limitations. First, not all isolates of $E$.coli BSI were included in the analysis; therefore, there may have been a selection bias. Second, we could not perform PFGE on E. coli isolated from the patients with early onset recurrent BSI. However, we postulated that most of early onset recurrent BSI were infected by identical strains. E. coli isolated from early onset recurrent BSI was highly resistant to third-generation cephalosporin or ciprofloxacin, which are most commonly used as initial therapy after identification of gram-negative BSI. Since initial empirical antibiotic therapy was not be effective, early 
onset recurrence might be caused by remnants of $E$. colithat had not been properly eliminated during the first episode of infection. Third, we showed that vascular catheter was associated with recurrent $E$. coli $\mathrm{BSI}$, as described in previous studies $[13,30]$. However, the number of patients with a vascular catheter was too low in our study. Therefore, the findings related to those few patients will need to be interpreted cautiously.

\section{Conclusion}

We found risk factors for recurrent $E$. coli $\mathrm{BSI}$, which may be helpful to healthcare providers in planning the patient follow-up after initial E. coli BSI episode. The detection of variable molecular feature in $E$. coli causing recurrent BSI suggests the need for an in-depth study to identify microbiological determinants associated with recurrent $E$. coli $\mathrm{BSI}$.

\section{Abbreviations}

BSI: bloodstream infection; ESBL: extended spectrum $\beta$-lactamase; PFGE: pulsed-field gel electrophoresis; ST 131: sequence type 131; WGS: whole genome sequencing

\section{Declarations}

\section{Ethics approval and consent to participate:}

The study protocol was approved by the Institutional Review Board (NHIMC-2019-01-018) of National Health Insurance Service Ilsan Hospital.

\section{Consent for publication:}

Not applicable.

\section{Availability of data and materials:}

All data generated or analyzed during this study are included in this published article (and its supplementary information files).

\section{Competing interests:}

The authors declare that they have no competing interests.

\section{Funding:}

This work was supported by the National Health Insurance Service llsan Hospital grant (No. NHIMC2019CR012 to YAK). The funder had no role in study design, data analysis, or preparation of the manuscript. 


\section{Authors' contributions:}

Conception and design of the study, YAK and YSP; acquisition of data, HC, YAK and YHS; analysis and interpretation of data, YCK and YAK; drafting the article, YCK; revising the article, HC, HL and KL; final approval, YAK and YSP

\section{Acknowledgements:}

none.

\section{References}

1. Kaper JB, Nataro JP, Mobley HLT. Pathogenic Escherichia coli. Nature Reviews Microbiology. 2004;2(2):123-40.

2. Bou-Antoun S, Davies J, Guy R, Johnson AP, Sheridan EA, Hope RJ. Descriptive epidemiology of Escherichia coli bacteraemia in England, April 2012 to March 2014. Euro Surveill. 2016;21(35).

3. Uslan DZ, Crane SJ, Steckelberg JM, Cockerill FR, 3rd, St Sauver JL, Wilson WR, et al. Age- and sexassociated trends in bloodstream infection: a population-based study in Olmsted County, Minnesota. Arch Intern Med. 2007;167(8):834-9.

4. Berger J, Diab-Elschahawi M, Blacky A, Pernicka E, Spertini V, Assadian O, et al. A matched prospective cohort study on Staphylococcus aureus and Escherichia coli bloodstream infections: extended perspectives beyond resistance. Am J Infect Control. 2010;38(10):839-45.

5. Bonten M, Johnson JR, van den Biggelaar AHJ, Georgalis L, Geurtsen J, de Palacios PI, et al. Epidemiology of Escherichia coli Bacteremia: A Systematic Literature Review. Clin Infect Dis. 2021;72(7):1211-9.

6. Wendt C, Messer SA, Hollis RJ, Pfaller MA, Wenzel RP, Herwaldt LA. Recurrent gram-negative bacteremia: incidence and clinical patterns. Clin Infect Dis. 1999;28(3):611-7.

7. Cattaneo C, Antoniazzi F, Tumbarello M, Skert C, Borlenghi E, Schieppati F, et al. Relapsing bloodstream infections during treatment of acute leukemia. Ann Hematol. 2014;93(5):785-90.

8. Marschall J, Doherty J, Warren DK. The epidemiology of recurrent Gram-negative bacteremia in a tertiary-care hospital. Diagn Microbiol Infect Dis. 2010;66(4):456-9.

9. Maslow JN, Mulligan ME, Arbeit RD. Recurrent Escherichia coli bacteremia. J Clin Microbiol. 1994;32(3):710-4.

10. Samet A, Sledzińska A, Krawczyk B, Hellmann A, Nowicki S, Kur J, et al. Leukemia and risk of recurrent Escherichia coli bacteremia: genotyping implicates E. coli translocation from the colon to the bloodstream. Eur J Clin Microbiol Infect Dis. 2013;32(11):1393-400.

11. Sanz-Garcia M, Fernandez-Cruz A, Rodriguez-Creixems M, Cercenado E, Marin M, Munoz P, et al. Recurrent Escherichia coli bloodstream infections: epidemiology and risk factors. Medicine (Baltimore). 2009;88(2):77-82. 
12. Woudt SHS, de Greeff SC, Schoffelen AF, Vlek ALM, Bonten MJM, Infectious Diseases Surveillance Information System-Antimicrobial Resistance Study G. Antibiotic Resistance and the Risk of Recurrent Bacteremia. Clin Infect Dis. 2018;66(11):1651-7.

13. Lee CH, Su LH, Chen FJ, Tang YF, Chien CC, Liu JW. Clinical and microbiologic characteristics of adult patients with recurrent bacteraemia caused by extended-spectrum $\beta$-lactamase-producing Escherichia coli or Klebsiella pneumoniae. Clin Microbiol Infect. 2015;21(12):1105.e1-8.

14. Charlson ME, Pompei P, Ales KL, MacKenzie CR. A new method of classifying prognostic comorbidity in longitudinal studies: development and validation. J Chronic Dis. 1987;40(5):373-83.

15. Horan TC, Andrus M, Dudeck MA. CDC/NHSN surveillance definition of health care-associated infection and criteria for specific types of infections in the acute care setting. Am J Infect Control. 2008;36(5):309-32.

16. CLSI. Performance Standards for Antimicrobial Susceptibility Testing. 27th ed. CLSI supplement M100. Wayne, PA: Clinical and Laboratory Standards Institute; 2017. In.

17. Kim H, Kim YA, Park YS, Choi MH, Lee GI, Lee K. Risk Factors and Molecular Features of Sequence Type (ST) 131 Extended-spectrum beta-Lactamase-producing Escherichia coli in Community-onset Bacteremia. Sci Rep. 2017;7(1):14640.

18. Johnson JR, Murray AC, Gajewski A, Sullivan M, Snippes P, Kuskowski MA, et al. Isolation and molecular characterization of nalidixic acid-resistant extraintestinal pathogenic Escherichia coli from retail chicken products. Antimicrob Agents Chemother. 2003;47(7):2161-8.

19. Welsh KJ, Skrobarcek KA, Abbott AN, Lewis CT, Kruzel MC, Lewis EM, et al. Predictors of relapse of methicillin-resistant Staphylococcus aureus bacteremia after treatment with vancomycin. J Clin Microbiol. 2011;49(10):3669-72.

20. Fowler VG, Jr., Kong LK, Corey GR, Gottlieb GS, McClelland RS, Sexton DJ, et al. Recurrent Staphylococcus aureus bacteremia: pulsed-field gel electrophoresis findings in 29 patients. J Infect Dis. 1999;179(5):1157-61.

21. Chang FY, Peacock JE, Jr., Musher DM, Triplett P, MacDonald BB, Mylotte JM, et al. Staphylococcus aureus bacteremia: recurrence and the impact of antibiotic treatment in a prospective multicenter study. Medicine (Baltimore). 2003;82(5):333-9.

22. King MD, Whitney CG, Parekh F, Farley MM. Recurrent invasive pneumococcal disease: a populationbased assessment. Clin Infect Dis. 2003;37(8):1029-36.

23. Lai CC, Hsu HL, Tan CK, Tsai HY, Cheng A, Liu CY, et al. Recurrent bacteremia caused by the Acinetobacter calcoaceticus-Acinetobacter baumannii complex. J Clin Microbiol. 2012;50(9):2982-6.

24. Hsu RB, Chen RJ, Chu SH. Risk factors for recurrent bacteremia in adult patients with nontyphoid salmonellosis. Am J Med Sci. 2004;328(6):315-8.

25. Thaden JT, Fowler VG, Sexton DJ, Anderson DJ. Increasing Incidence of Extended-Spectrum $\beta$ Lactamase-Producing Escherichia coli in Community Hospitals throughout the Southeastern United States. Infect Control Hosp Epidemiol. 2016;37(1):49-54. 
26. Rodríguez-Baño J, Picón E, Gijón P, Hernández JR, Ruíz M, Peña C, et al. Community-onset bacteremia due to extended-spectrum beta-lactamase-producing Escherichia coli: risk factors and prognosis. Clin Infect Dis. 2010;50(1):40-8.

27. Colpan A, Johnston B, Porter S, Clabots C, Anway R, Thao L, et al. Escherichia coli sequence type 131 (ST131) subclone $\mathrm{H} 30$ as an emergent multidrug-resistant pathogen among US veterans. Clin Infect Dis. 2013;57(9):1256-65.

28. Grant MA, Weagant SD, Feng P. Glutamate decarboxylase genes as a prescreening marker for detection of pathogenic Escherichia coli groups. Applied and environmental microbiology. 2001;67(7):3110-4.

29. Fröding I, Hasan B, Sylvin I, Coorens M, Nauclér P, Giske CG. Extended-Spectrum- $\beta$-Lactamase- and Plasmid AmpC-Producing Escherichia coli Causing Community-Onset Bloodstream Infection: Association of Bacterial Clones and Virulence Genes with Septic Shock, Source of Infection, and Recurrence. Antimicrob Agents Chemother. 2020;64(8).

30. Hanna H, Afif C, Alakech B, Boktour M, Tarrand J, Hachem R, et al. Central venous catheter-related bacteremia due to gram-negative bacilli: significance of catheter removal in preventing relapse. Infect Control Hosp Epidemiol. 2004;25(8):646-9.

\section{Figures}

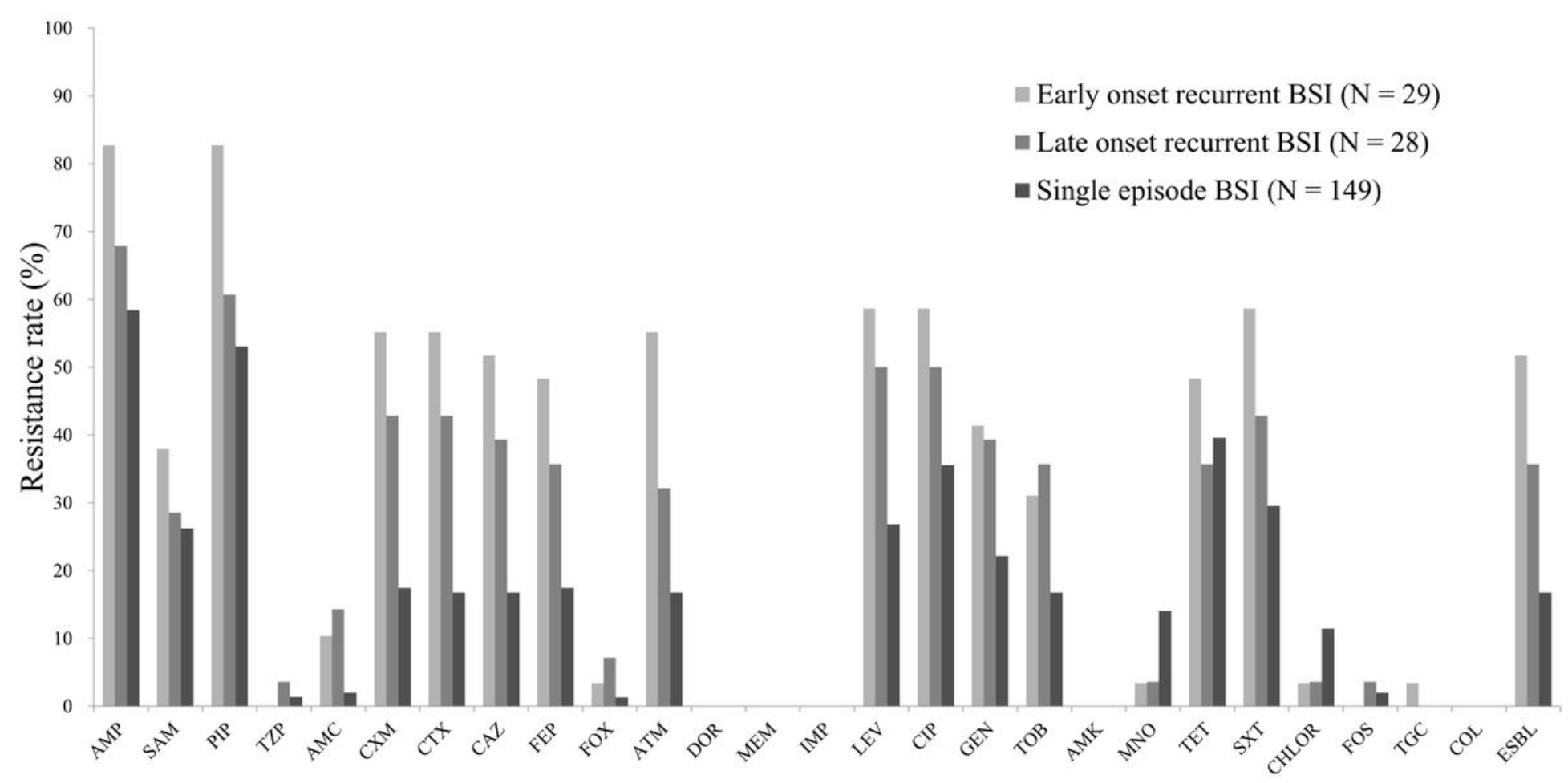

Figure 1 
Antimicrobial resistance rates of Escherichia coli isolated from patients with bloodstream infection ( $N=206)$. Abbreviation: BSI, bloodstream infection; Amp, ampicillin; Sam, ampicillin/sulbactam; Pip, piperacillin; Tzp, piperacillin/tazobactam; Amc, amoxicillin/clavulanic acid; Cxm, cefuroxime; Ctx, cefotaxime; Caz, ceftazidime; Fep, cefepime; Fox, cefoxitin; Atm, aztreonam; Dor, doripenem; Mem, meropenem; Imp, imipenem; Lev, levofloxacin; Cip, ciproflroxacin; Gen, gentamicin; Tob, tobramycin; Amk, amikacin; Mno, minocycline; Tet, tetracycline; Sxt, cotrimoxazole; Chlor, chlorampenicol; Fos, fosfomycin; Tgc, tigecycline; Col, colistin; ESBL, extended spectrum $\beta$-lactamase

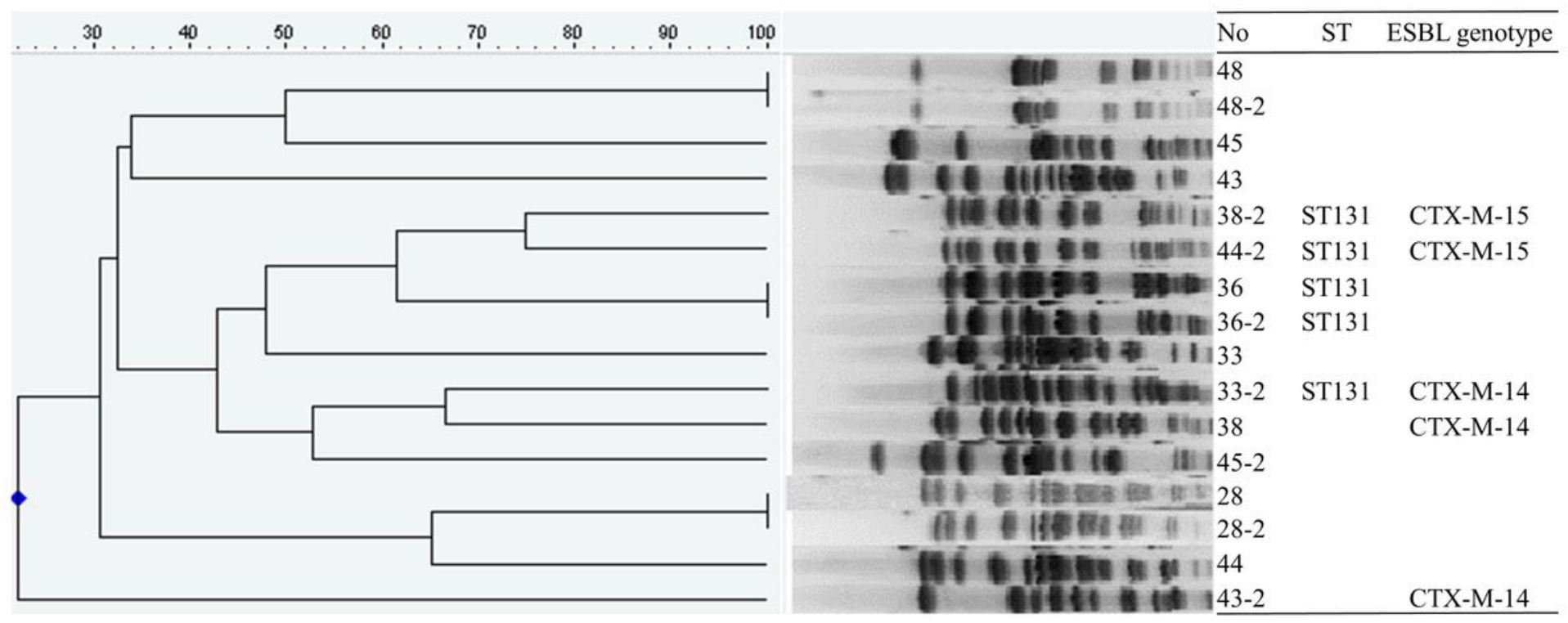

Figure 2

Dendrogram of Xbal-restricted DNA of Escherichia coli isolated from late onset recurrent bloodstream infection ( $\mathrm{N}=107)$. Pulsed-field gel electrophoresis was performed with size marker, Lambda Ladders (Promega, Fitchburg, WI, USA). Abbreviation: ST, sequence type; ESBL, Extended spectrum $\beta$-lactamase

\section{Supplementary Files}

This is a list of supplementary files associated with this preprint. Click to download.

- Supplementarymaterial.docx 